\title{
Rehabilitation Strategy for Post-stroke Recovery using an Innovative Elbow Exoskeleton
}

\author{
Soumya K. Manna, Venketesh N. Dubey
}

Faculty of Science \& Technology, Bournemouth University, United Kingdom, BH12 5BB

Intensive and adaptive rehabilitation therapy is beneficial for post-stroke recovery. Three modes of rehabilitation are generally performed at different stages after stroke; external force based control in the acute stage, assistive force based rehabilitation in the midway of recovery and resistive force based rehabilitation in the last stage. To achieve the above requirements, an innovative elbow exoskeleton has been developed to incorporate the three modes of rehabilitation in a single structure. The structure of the exoskeleton has been designed in such a way that the whole working region is divided into three where each region can provide a different mode of rehabilitation. Recovery rate can be varied for individuals since it depends on various parameters. To evaluate the rate of recovery, three joint parameters have been identified: range of angular movement, angular velocity and joint torque. These parameters are incorporated into the framework of planning a novel rehabilitation strategy which is discussed in this paper along with the structural description of the designed exoskeleton.

Nomenclatures
$R I_{t}=$ Rate of recovery
$H I_{t}=$ Health index
$\beta=$ Elbow joint angle/range
$\omega=$ Angular frequency of elbow joint
$L_{m}=$ Change of load carrying capacity
$P_{l}=$ Load carrying capacity of the joint
$T=$ Time over a period of testing
$t=$ Time to compute one rotation
$n=$ Number of turns of motor
$\theta=$ Motor rotation angle
$\dot{\theta}=$ Angular velocity of the motor
$V=$ Linear velocity of the nut slider
$x=$ Position of the nut slider
$M=$ Mass of the forearm and supporting link
$L_{1}=$ Distance from the elbow joint to the centre
of gravity of forearm and the supporting link
$p=$ Pitch of the leadscrew

\section{Nomenclatures}

$R I_{t}=$ Rate of recovery

index

$\omega=$ Angular frequency of elbow joint

$L_{m}=$ Change of load carrying capacity

$P_{l}=$ Load carrying capacity of the joint

$T=$ Time over a period of testing

$t=$ Time to compute one rotation

$n=$ Number of turns of motor

$\theta=$ Motor rotation angle

$\dot{\theta}=$ Angular velocity of the motor

$V=$ Linear velocity of the nut slider

$x=$ Position of the nut slider

$L_{1}=$ Distance from the elbow joint to the centre

$p=$ Pitch of the leadscrew

\section{Introduction}

In the last two decades, the mortality rate due to stroke has increased as per the statistics from WHO (World Health Organization) ${ }^{1}$. Lack of sufficient number of caregivers and expensive manual treatment have made robotic rehabilitation $d_{1}=$ Pitch diameter of the leadscrew

$\varphi=$ Friction angle

$\delta=$ Lead angle of the leadscrew

$\alpha=$ Angle between connecting rod and slider

$r=$ Length of the crank

$K_{2}=$ Stiffness of $\mathrm{S}_{2}$

$x_{s 2}=$ Free length of $S_{2}$

$f=$ Joint frequency

$\tau_{l}=$ Total torque

$\tau_{p}=$ Joint torque from patient

$\tau_{D}=$ Desired joint torque

$r_{1}=$ Radius of the pulley at the elbow joint

$K_{3}=$ Stiffness of $\mathrm{S}_{3}$

$x^{\prime}{ }_{s 3}=$ Free length of $\mathrm{S}_{3}$

$K_{4}=$ Stiffness of $\mathrm{S}_{4}$

$x^{\prime}{ }^{\prime} 4=$ Free length of $S_{4}$

$K^{\prime}=$ Joint stiffness a promising choice for recovery of post-stroke patients ${ }^{2}$. Robotic rehabilitation for upper arm was started with end-effector based systems such as MIT MANUS $^{3}$ which provided exercise by holding the distal end of the manipulator; specific 
joint oriented exercises are not feasible using endeffector based systems. Unsupported arm resulted in rehabilitation inefficiency on end-effector based system that drove the development of exoskeleton based on the shape of human body. Most of the exoskeletons designed for rehabilitation are used for a specific type of training which are either providing assistive or resistive force to users. However, before selecting the type of exoskeleton suitable for post-stroke rehabilitation, it is necessary to know the type of exercises required to fit the optimal rehabilitation training. A series of exercise modules are followed by different organizations $^{1}$ providing exercises for stroke patients. It may produce normal arm movements as well as specific joint based training to enhance the motor functions. There is no standard approach documented yet for providing specific exercise from acute stage to full recovery stage.

After surveying several published guidelines ${ }^{4,5,6}$, the full exercise module can be divided into three stages for post-stroke rehabilitation. In acute stage, external force is applied for rehabilitation because patients are unable to move their joints themselves. The neurological motor function improves with time to initiate the joint movement. Assistive force is helpful at this stage to support such patients. At the start, the level of assistance is higher but the amount of assistance is reduced gradually as the patient gains more strength with time. This support improves joint coordination and patients are capable of meeting their daily activities. To enhance the muscle strength and joint torque, exercises are normally designed to place the joint motions in more difficult situations where patients need more joint torque to achieve the goal. Such exercises help patients to enhance their load bearing capability.

Integration of the three stages of rehabilitation in a single structure can possibly be accomplished in two ways; one is software-based solution and the other is mechanical approach. In software-based control, it is possible to generate a wide range of torque using adaptive algorithms ${ }^{7}$, however, the

1 www.saebo.com, www.flintrehab.com, www.strokerehab.com functionality of those systems is reliant on the sensors employed ${ }^{8,9,10}$. The adaptive control can take over the performance of rehabilitation task and reduce patient's participation ${ }^{11}$. Joint spasticity can generate unwanted signals which can create adverse effect on joint ${ }^{12}$. It also has few other limitations such as use of actuator results in constant draining of energy which increases the size of energy source.

A few hardware-based exoskeletons have been developed to incorporate different modes of rehabilitation but the use of those systems is only limited to hand rehabilitation such as HANDEXOS $^{13}$. Engaging more than one actuator in a single system can generate different modes of rehabilitation; however, it will make the system bulky. Spring-based exoskeleton can only generate assistive force ${ }^{14}$ therefore, not suitable for providing the adaptive exercises.

In order to overcome these shortfalls, passive elements such as spring can be combined with an electric motor which can produce three modes of rehabilitation in an exoskeleton. After evaluating the properties and working principle of many existing exoskeletons ${ }^{15}$, we have come up with a novel design to provide three modes of rehabilitation in a single exoskeleton.

\section{Design of the mechanism}

In this exoskeleton, the mode of rehabilitation can be changed based on patients' health status and the level of recovery. The entire operating region is partitioned into three sections. The first section controls the joint movements externally using electric motor without patient's participation whereas the middle section allows patients to do joint movements supported by the spring force and the range of assistance can be varied by adjusting the displacement of the spring. In the last section, a spring assembly is used to provide variable resistive force to the joint. With this strategy, the energy source is only used in the first rehabilitation mode to power the motor whereas the other two modes can work without any power supply on the stored energy of springs. The switching between two rehabilitation modes is also achieved using springs. An integrated 
rehabilitation strategy is designed with different exercises consisting of a variable range of angular movement, velocity and load. These three joint parameters (joint angle, velocity and torque) are also used as the key parameters to measure the recovery rate of patients. The $3 \mathrm{D}$ model of the designed elbow exoskeleton is shown in Fig. 1.

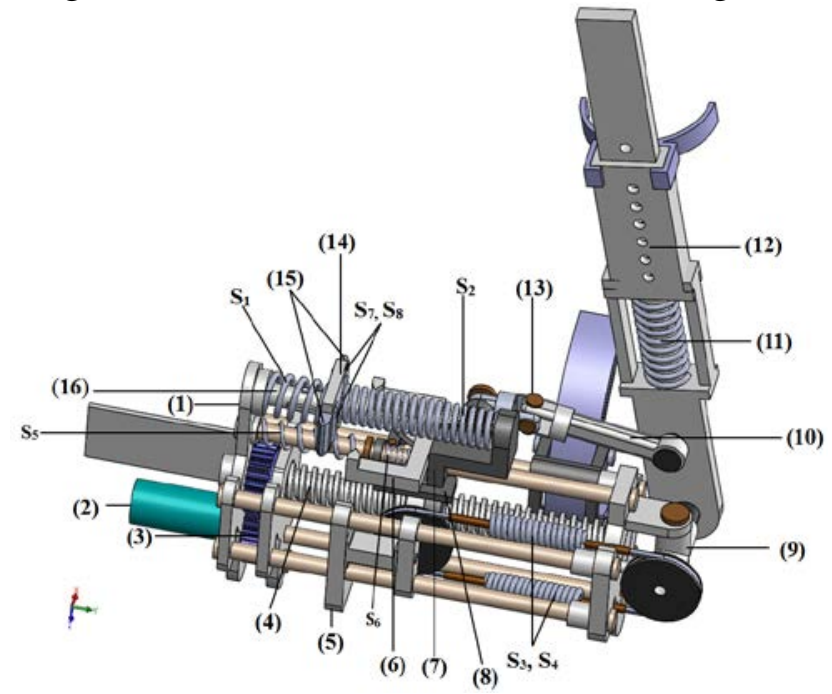

Figure 1. 3D model of the exoskeleton

\begin{tabular}{|ll|}
\hline (1) Baseplate & (11) Compression spring \\
(2) Motor & (12) Forearm supporting link \\
(3) Gear transmission & (13) Universal joint \\
(4) Leadscrew & (14) Rectangular slider \\
(5) Slider for variable stiffness & (15) Connected plates \\
(6) Nut slider & (16) Small cylindrical rod \\
(7) Concentric slider & $\mathrm{S}_{1}, \mathrm{~S}_{5}, \mathrm{~S}_{6}=$ Compression springs \\
(8) Claw type jaws & $\mathrm{S}_{2}, \mathrm{~S}_{3}, \mathrm{~S}_{4}=$ Extension springs \\
(9) Elbow joint & $\mathrm{S}_{7}$ and $\mathrm{S}_{8}=$ Torsional springs \\
(10) Connecting rod & \\
\hline
\end{tabular}

Fig. 2 shows the developed prototype of the elbow exoskeleton. All mechanical parts have been

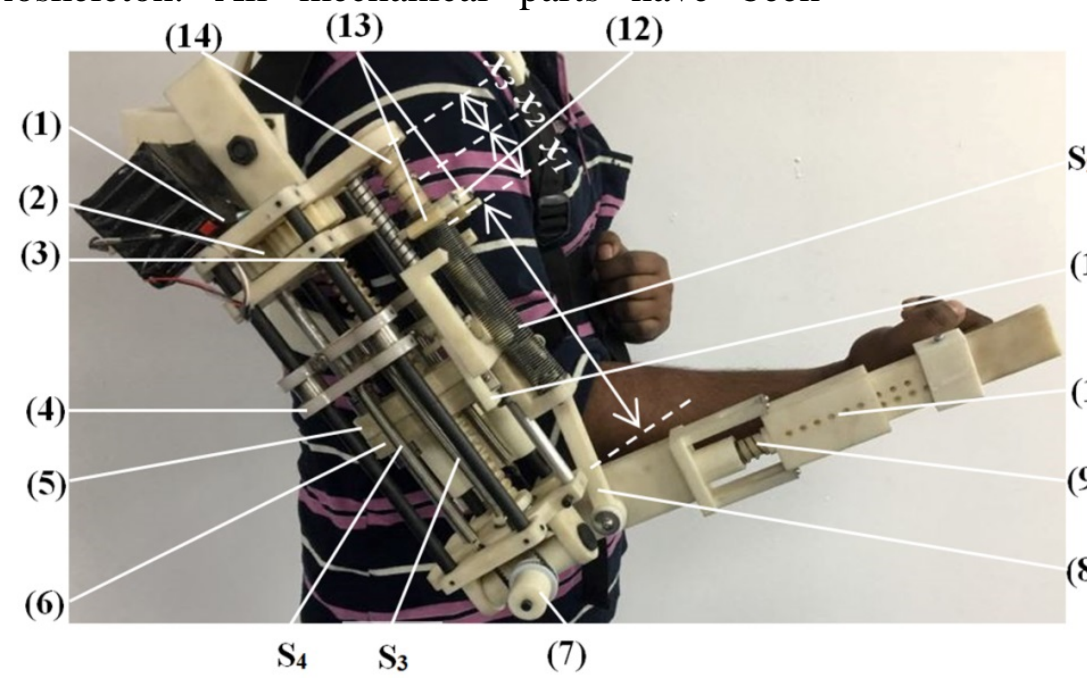

Figure 2. Prototype of the developed exoskeleton

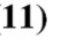

(10) fabricated by 3D printing. All rotational and sliding contacts have been designed with a bearing to reduce the frictional loss. ABS (Acrylonitrile butadiene styrene) is used as structural material for its hardness and lightweight. The exoskeleton provides all required exercises for three different stages of rehabilitation. These stages refer to three rehabilitation regions in the exoskeleton which are connected to each other and come one after another. The exoskeleton can produce modes of rehabilitation aligned with the post-stroke recovery stages. In the designed elbow exoskeleton, the actuation system of each rehabilitation stage is customized and integrated into a single module to meet the specific requirements of post-stroke recovery stages as shown in Fig. 3. In the first region (acute stage), user's joint is actuated by the motor since patients do not have enough muscle strength in this phase. In the mid-stage, the displacement of $\mathrm{S}_{2}$ is changed in a way to provide assistive force to the user and in the last region of operation; $\mathrm{S}_{3}$ and $\mathrm{S}_{4}$ are extended to create the resistive force for the last stage of recovery. A single motor is used to achieve all these functions with the help of springs. Spring based switching mechanism removes the complexity of electromechanical switches. The actuation system of each rehabilitation mode is described in detail in the following paragraphs.
(1) Motor
(2) Gear transmission
(3) Leadscrew
(4) Slider for variable stiffness
(5) Nut slider
(6) Concentric slider
(7) Elbow joint
(8) Connecting rod
(9) Compression spring
(10) Forearm supporting link
(11) Claw type jaws
(12) Rectangular slider
(13) Connected plates
(14) Small cylindrical rod 


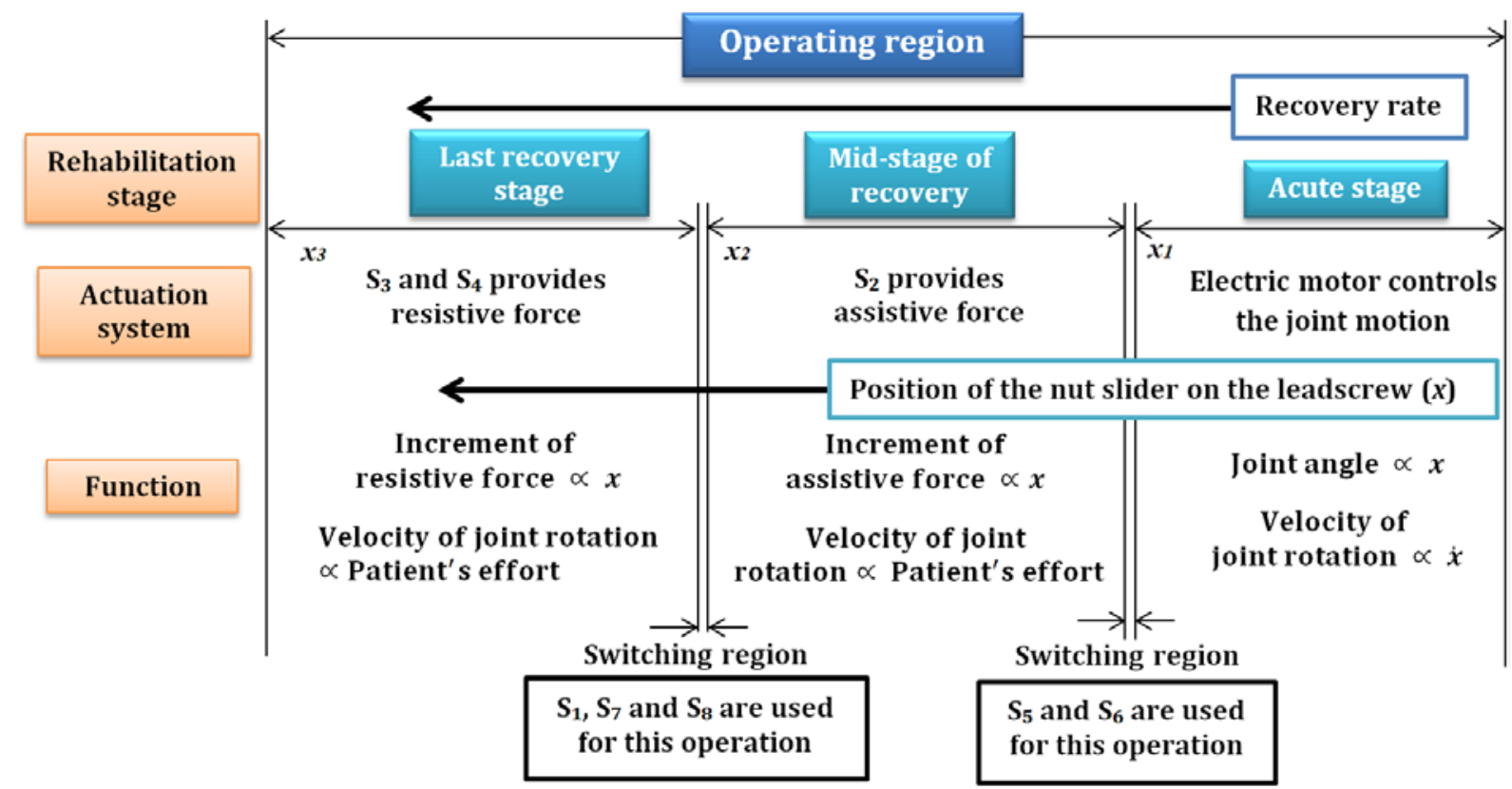

Figure 3. Rehabilitation modes based on operating regions of the exoskeleton

A leadscrew is used to transfer motion from the motor to the elbow joint. Two types of sliders are used in the leadscrew; one of which is the nut slider which follows the guiding path of the leadscrew and the other is concentric to the leadscrew which is free to move over the leadscrew. The region of operation is chosen by the position of the nut slider on the leadscrew $(x)$ hence the mode of rehabilitation is changed as:

- Motor controlled mode

$$
\begin{aligned}
& 0 \leq x \leq x_{1} \\
& x_{1}<x \leq x_{2} \\
& x_{2}<x \leq x_{3}
\end{aligned}
$$

$\left(x_{1}, x_{2}\right.$ and $x_{3}$ are the switching positions of the nut slider.)

In the motor controlled mode, the two sliders are attached together by claw type of jaws which are energised by an extension spring $\left(\mathrm{S}_{6}\right)$ hence work like a single unit (Fig. 4a). Clockwise rotation of the motor pushes the nut slider in the forward direction as a result, the motor actuates the elbow joint. When the nut slider crosses the first operating region $\left(x>x_{1}\right)$, two compression springs $\left(\mathrm{S}_{5}\right.$ and $\mathrm{S}_{6}$ ) push each other and the jaws are unlocked due to the higher spring force from $\mathrm{S}_{5}$ (Fig. 4b).

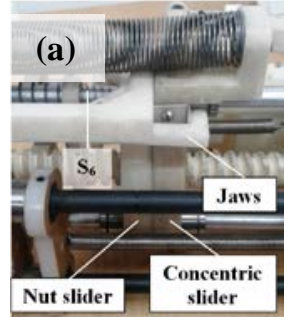

(b)

Figure 4. Switching from motor controlled to assistive mode

Then comes the assistive mode, here the stiffness of spring $\left(\mathrm{S}_{2}\right)$ provides assistive force (Fig. 5a) during flexion to help the user in arm lifting. The same spring $\left(\mathrm{S}_{2}\right)$ resists arm freefall during extension. In order to switch from assistive to resistive mode, the nut slider pushes the rectangular slider connected to $S_{2}$. In this region $\left(x_{1}<x \leq x_{2}\right)$, the range of assistive force can be varied by changing the starting position of $\mathrm{S}_{2}$. At the end of this region, both torsional springs $\left(S_{7}\right.$ and $\mathrm{S}_{8}$ ) are deflected (Fig. 5b) and the rectangular slider returns to the original position due to the compressive force from $\mathrm{S}_{1}$ (Fig. 5a).

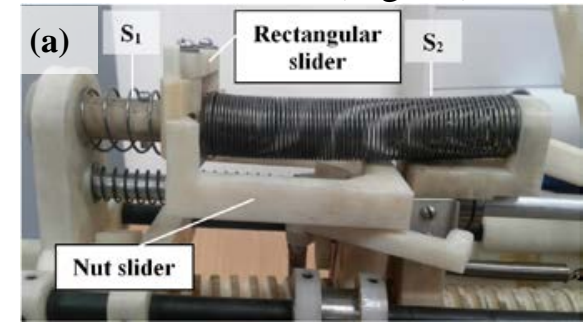




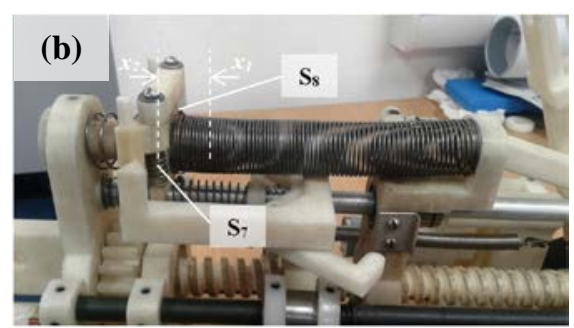

Figure 5. Switching from assistive to resistive mode

In the resistive rehabilitation mode, stretching of two extension springs $S_{3}$ and $S_{4}$ (Fig. 6) changes the joint stiffness in the region $\left(x_{2}<x \leq x_{3}\right)$ due to pushing of the nut slider to another slider connected to $S_{3}$ and $S_{4}$, thus increases the resistive force. The required level of stiffness of the spring is decided by the weight of the forearm.

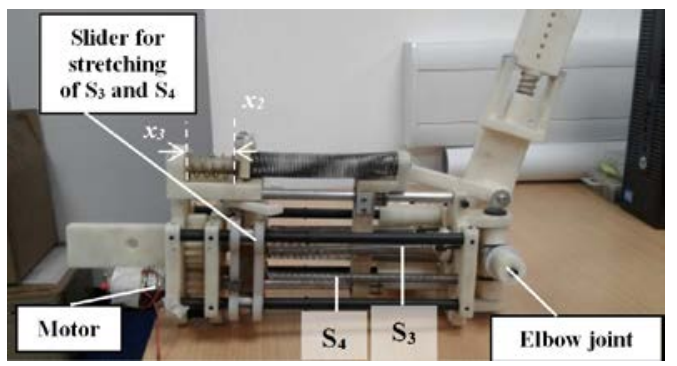

Figure 6. Springs used in resistive mode

A universal joint is used at the elbow joint to improve the comforts level by allowing lateral movements. To maintain the alignment of the centre of rotation between the exoskeleton and user, the forearm has a passive translational joint with a compression spring whose length is varied to match the forearm length (Fig. 2). The length of the forearm supporting link can also be adjusted for different user.

The whole structure of elbow exoskeleton is attached to user's body using shoulder support to offload the weight on user's arm. The structure is made of circular holders and is placed around the body with belt tightening. It supports from two sides; two circular holders are fitted around the chest connected by a solid link and help to attach the exoskeleton with user's body in transverse plane. The shoulder holder takes care of the weight of the exoskeleton against gravity. The supporting structure is assembled by detachable components, therefore the length of formation around the chest and shoulder can be changed as per different body shape requirements as shown in Fig. 7.

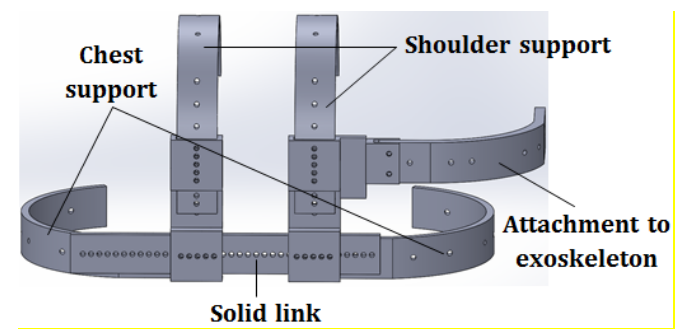

a. 3D model

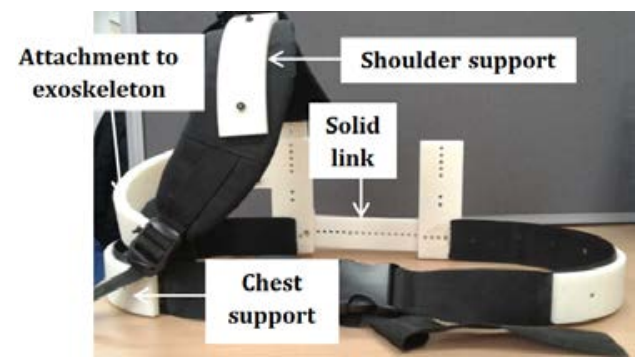

b. Actual prototype

Figure 7. Design of the supportive arm structure

\section{Rehabilitation strategy}

Rehabilitation strategy is planned as the series of exercises required in post-stroke recovery process. It depends on two parameters; one is the structural parameters (dimension of the mechanical components, stiffness of springs, motor torque) which decide the working range of the mechanical parts. The other is the clinical parameters which decide rehabilitation mode and its outcome. After analyzing the rehabilitation protocol with the design specifications of the developed exoskeleton, an adaptive exercise module is proposed to accommodate the three modes of rehabilitation.

The ability of a human joint can be evaluated based on its joint angle range, velocity and torque bearing capacity because those three parameters show the reachable points, frequency of movement and weight lifting capacity. Therefore, these three joint parameters are considered in this exoskeleton to control the mode of rehabilitation and also used to evaluate the post-stroke recovery rate. The rate of recovery $\left(R I_{t}\right)$ is an iterative process which can be evaluated by measuring the difference between present and past health index 
$\left(H I_{t}\right)$ which quantifies the joint condition based on joint angle $(\beta)$, angular frequency of the joint $(\omega)$ and change in load carrying capacity $\left(L_{m}\right)$.

$$
\begin{aligned}
& R I_{t}=\frac{H I_{t}-H I_{t-1}}{T}\left[H I_{t} \rightarrow f\left(\beta, \omega, L_{m}\right)\right] \\
& \omega=\frac{\beta(t)-\beta(t-1)}{t} \\
& L_{m}=P_{l}(t)-P_{l}(t-1)
\end{aligned}
$$

The exoskeleton offers three stages of rehabilitation in such a way that the relationship between the recovery rate and the mode of rehabilitation is directly associated. It is recommended that the boundary condition of the recovery index for switching from one mode of rehabilitation to another is determined by the physiotherapist. If patients can cross the boundary, they will be ready to transfer from one mode of rehabilitation to another. The control algorithm will automatically put the nut slider in a specific position required for this.

\subsection{Electric motor controlled mode}

Considering spasticity and joint stiffness, it is not suitable to provide a full range of motion to the affected joint at the starting phase. The joint angle and velocity of the joint movement should be increased gradually. The elbow flexion-extension is achieved by the reciprocating motion of the nut slider due to motor rotation. If the number of rotations of the motor is increased, the rotation angle of the elbow is simultaneously increased provided the nut slider is in the first region. On the other hand, different speed of the motor changes the velocity of the joint movement. The load is fixed in this mode of rehabilitation.

As both leadscrew based sliders act as a single attachment during the rehabilitation, the rotation angle $(\beta)$ of the elbow joint is a function of $x$ (position of nut-slider), thus depending on the motor rotations $(n, \theta)$.

i.e. $(n, \theta) \rightarrow(x) \rightarrow \beta$

Therefore, $(n, \dot{\theta}) \rightarrow(V) \rightarrow \omega$
In the first rehabilitation mode, the region covered by the electric motor $\left(0 \leq x \leq x_{1}\right)$ is equivalent to the full range of motion of the elbow joint. Therefore, the two claw type jaws combine the two sliders as a single unit until the joint takes a full rotation. Beyond this region, the jaws will automatically open and change the mode of rehabilitation where the joint torque is provided by spring force. In the designed mechanism, the range of the nut slider in this rehabilitation mode is 0 to $0.18 \mathrm{~m}$ which can generate a rotation angle of $135^{\circ}$ at the elbow joint. The torque analysis of the leadscrew based sliding motion can be derived from the leadscrew mechanism ${ }^{16}$ (Fig. 8).

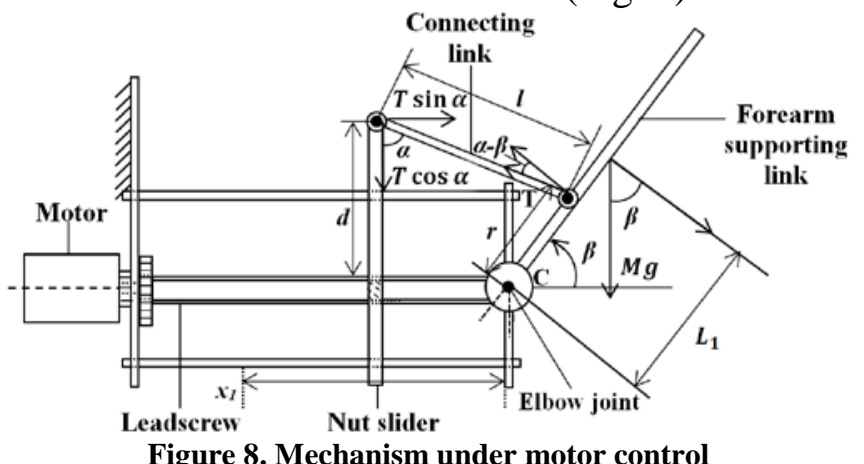

Considering the frictional force between the nut slider and leadscrew, the required motor torque $(\tau)$ can be given by ${ }^{16}$

$\tau=\frac{M g L_{1} \cos \beta(\cos \alpha+\sin \alpha \tan (\delta-\varphi)) d_{1}}{2 r \cos (\alpha-\beta)}$

$\tan \delta=\frac{p}{\pi d_{1}}$

Where,

The weight of the forearm can be varied for different users. A range of the forearm mass (1 kg to $3 \mathrm{~kg}$ ) has been considered and the required motor torque level is shown in Fig. 9.

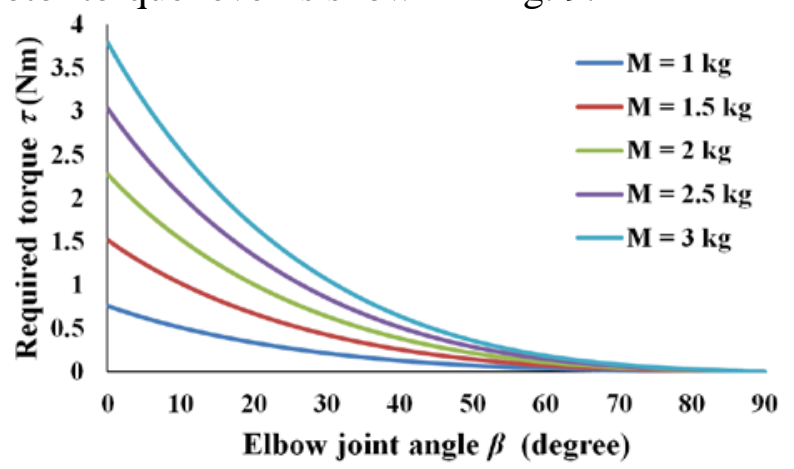

Figure 9. Motor torque for different forearm mass 
Continuous movement of the motor in clockwise and anticlockwise direction with different angle and speed improves the joint rehabilitation. In this rehabilitation mode, the number of repetitions ( $\mathrm{n} 1$ and n2) for each stage as well as the incremental step size of rotation $\left(\theta_{1}\right)$ and angular velocity $\left(\dot{\theta}_{2}\right)$ is determined by the physiotherapist to maintain the steady rehabilitation process (Fig. 10).

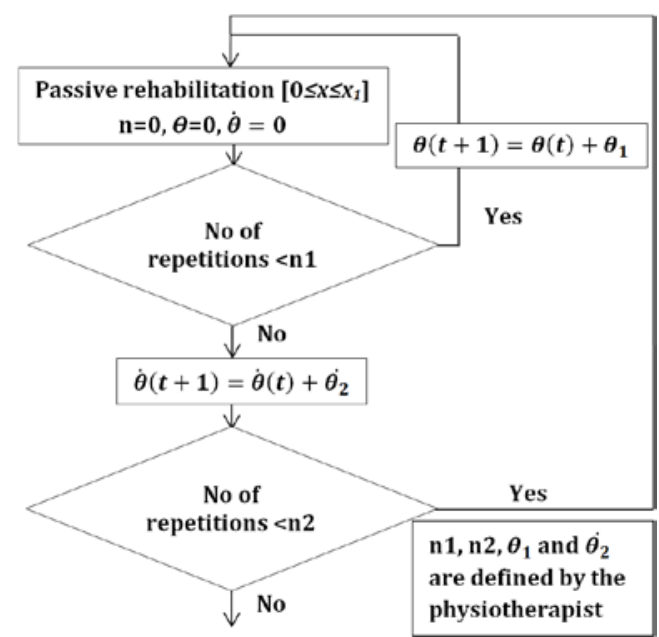

Figure 10. Rehabilitation parameters in motor controlled mode

\subsection{Assistive mode}

Patient's effort is the most important factor in this rehabilitation mode. When the nut slider and concentric slider are detached from each other, users can rotate the elbow joint themselves and the supporting force is provided by an extension spring $\left(\mathrm{S}_{2}\right)$ as shown in Fig. 11.

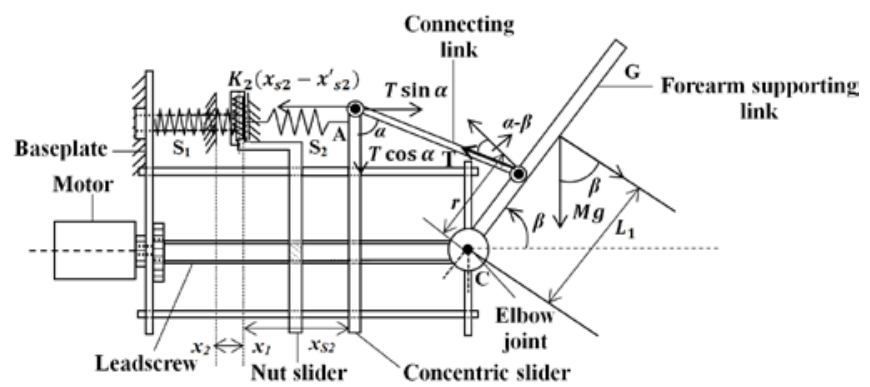

Figure 11. Generation of the assistive force using springs

The unlocking condition allows the concentric slider to move freely on the leadscrew in this region. As the concentric slider is connected to the elbow joint using connecting link, therefore the position of the concentric slider varies with elbow joint angle. $x_{s 2} \rightarrow f(\beta)$

In this mode the required torque $\left(\tau^{\prime}\right)$ for rotating the elbow joint is given by

$\tau^{\prime}=M g L_{1} \cos \beta$

The assistive force provided by $\mathrm{S}_{2}$ is

$f_{s 2}=K_{2}\left(x_{s 2}-x_{s 2}^{\prime}\right)$

After calculating the force equilibrium at point A (Fig. 11), the stiffness of $S_{2}\left(K_{2}\right)$ is given by ${ }^{16}$

$K_{2}=\frac{M g L_{1} \cos \beta \sin \alpha}{r \cos (\alpha-\beta)\left(x_{s 2}-x \prime_{s 2}\right)}$

The value of $K_{2}$ is calculated for different weight of forearm (1 to $3 \mathrm{~kg}$ ) which shows that $K_{2}$ can be varied from $246.55 \mathrm{~N} / \mathrm{m}$ to $493.10 \mathrm{~N} / \mathrm{m}$. The value of $K_{2}$ should be high enough to cover a range of patients.

The range of assistive force for an individual can be increased by changing the range of $x_{s 2}$. The backward movement of the nut slider for switching from assistive mode to resistive mode will shift the starting point of $S_{2}$ and the range of $x_{s 2}$ will be increased, thus providing more assistive force as shown in Fig. 12. In this exoskeleton, the maximum range of the assistive force will be provided if the position of nut slider will be at the end of this region. The range of displacement of $S_{2}$ can be varied from $0.12 \mathrm{~m}$ to $0.14 \mathrm{~m}$ for the position of the nut slider from $0.181 \mathrm{~m}$ to $0.2 \mathrm{~m}$.

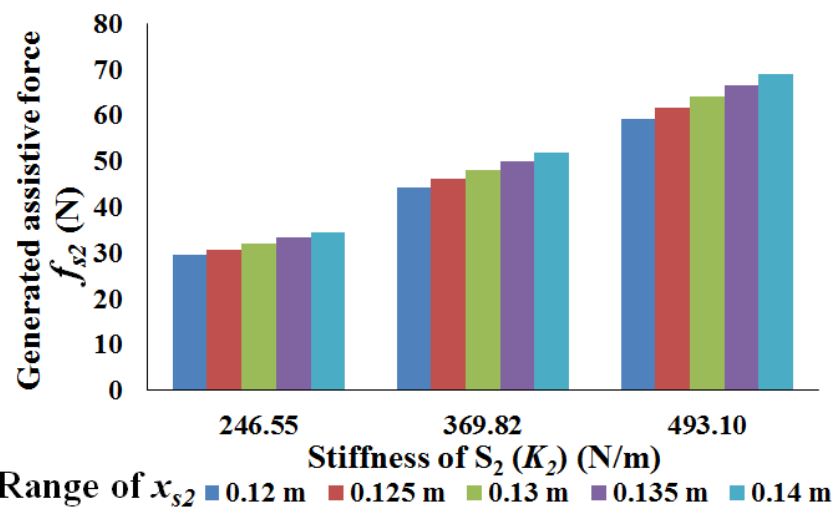

Figure 12. Variation of $f_{s 2}$ with the range of displacement $\left(x_{s 2}\right)$

The percentage of user's participation in rehabilitation task is defined as the recovery rate of the patient. Higher the percentage of 
participation better is the recovery rate. Normally, the assistive spring force should be kept lower than the desired joint torque so that patients endeavour to achieve it by giving more labour, thus they will practice gaining more strength to their joint. Based on the recovery status, the position of the nut slider should come forward to engage with less assistive force to encourage patients to put more effort. Theoretically, the position of the nut slider should be increased for giving more assistive force to patients if they are unable to move their joints. However, this strategy is applicable at the beginning when patients are initiating active joint movements, later the assistive force is decreased when patients can move their joints easily, just to motivate them to put extra effort.

Load sharing capacity of a patient is defined by

$$
P_{l}=\frac{\tau_{p}}{\tau_{l}} \times 100
$$

During the assistive mode, the decrement of the distance travelled by the nut slider will be inversely proportional to the difference between the desired torque $\left(\tau_{D}\right)$ and the present joint torque provided by the patient $\left(\tau_{p}\right)$. This rehabilitation mode is represented in flow diagram, Fig. 13.

$\Delta x \propto \frac{1}{\left(\tau_{D}-\tau_{p}\right)}$

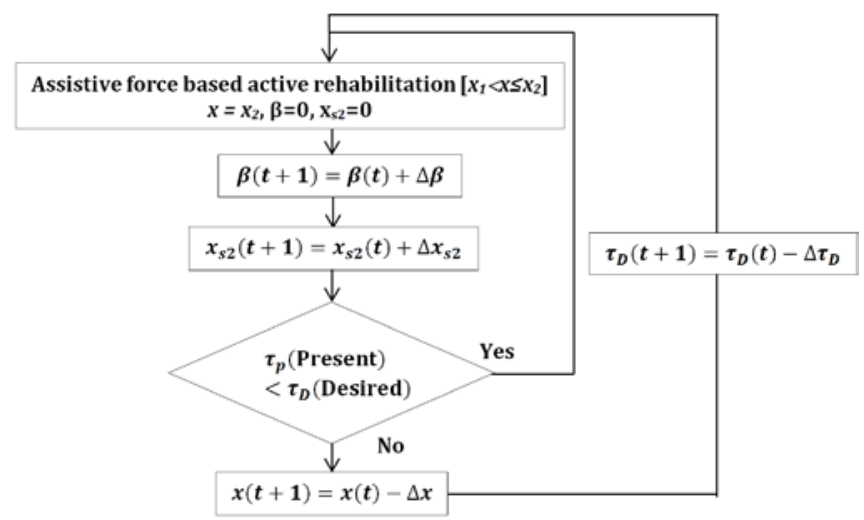

Figure 13. Parameters of assistive rehabilitation mode

\subsection{Resistive mode}

In this mode, a variable resistive force is applied to the elbow joint in terms of changing the joint stiffness (Fig. 14). This mode of rehabilitation is equivalent to the exercise with a variable load where the difficulty level in therapy is increased with time.

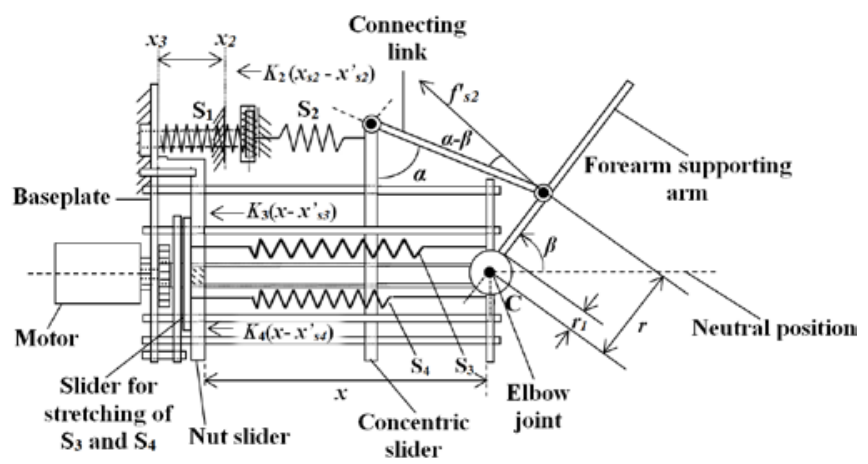

Figure 14. Generation of resistive force in the exoskeleton

In this mode, the stiffness of elbow joint is dependent on the stiffness of $S_{2}, S_{3}$ and $S_{4}$. In this configuration, the joint stiffness can be taken as a function of the distance travelled by the nut slider $(x)$ and elbow joint angle $(\beta)$. Fig. 15 shows the variation of joint stiffness for the distance travelled by the nut slider in this rehabilitation mode. The position of the nut slider can be varied from $0.201 \mathrm{~m}\left(x_{2}\right)$ to $0.24 \mathrm{~m}\left(x_{3}\right)$ during this rehabilitation regime.

As shown in Fig. 14,

$f^{\prime}{ }_{s 2}=\frac{K_{2}\left(x_{s 2}-x_{s 2}\right) \cos (\alpha-\beta)}{\sin (\alpha)}$

The stiffness of the elbow joint is same as the torsional stiffness $K^{\prime}$ which is given by.

$$
\begin{aligned}
& K^{\prime}= \\
& \frac{2 r_{1}\left\{\left(K_{4}-K_{3}\right) x-\left(K_{3} x \prime_{s 3}-K_{4} x \prime_{s 4}\right)\right\}-\frac{K_{2} r\left(x_{s 2}-x_{s 2}^{\prime}\right) \cos (\alpha-\beta)}{\sin \alpha}}{\beta}+ \\
& 2 r_{1}^{2}\left(K_{4}+K_{3}\right)
\end{aligned}
$$

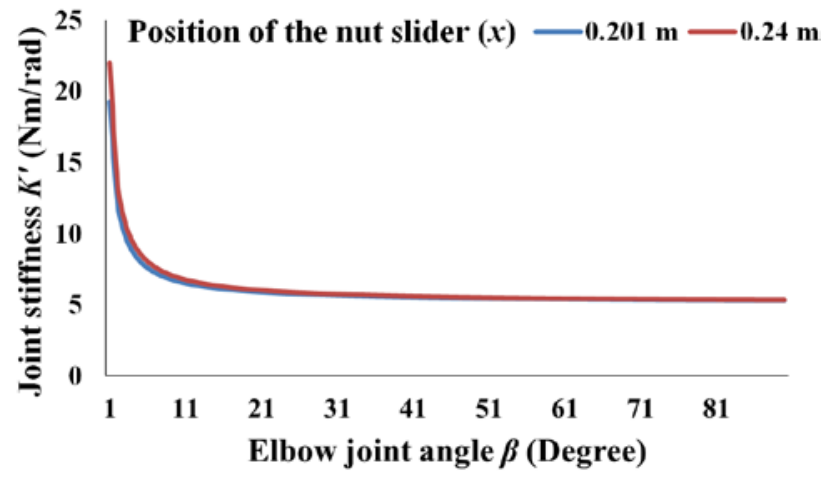

Figure 15. Elbow joint stiffness with nut slider position 
The required joint torque of elbow will increase with the joint stiffness.

Therefore, $\tau_{p} \propto K^{\prime}$

In this rehabilitation mode, $\tau_{p}=\tau_{l}$

If patients can overcome the resistive force by engaging with more joint torque, it shows the sign of recovery. In this mode of rehabilitation, the increment of the distance $(\Delta x)$ traveled by the nut slider is proportional to the difference between the desired torque $\left(\tau_{D}\right)$ and the present joint torque $\left(\tau_{p}\right)$ shown in Fig. 16.

Therefore $\Delta x \propto\left(\tau_{D}-\tau_{p}\right)$

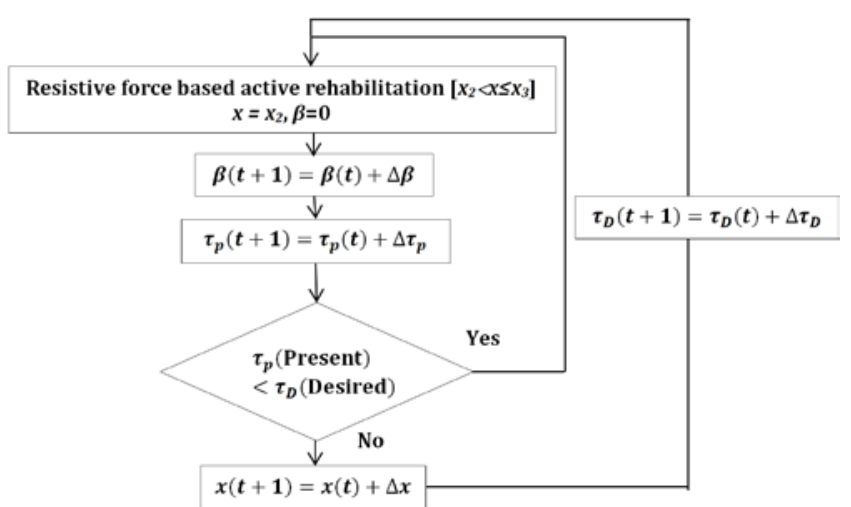

Figure 16. Parameters in resistive rehabilitation mode

\section{Electronic circuit}

All components are interfaced with Arduino Uno board (Operating voltage: $5 \mathrm{~V}$, RAM: $2 \mathrm{~KB}$, Clock Speed: $16 \mathrm{MHz}$ ) for collecting the joint parameters and controlling exercises (Fig. 17). A DC motor (Model: ZYTD520, Weight: $0.210 \mathrm{~kg}$, Power: $5 \mathrm{~W}$ ) is used for moving the nut slider on the leadscrew. The motor is operated at $12 \mathrm{~V}$ and it can provide maximum torque up to $4.6 \mathrm{Nm}$. Arduino Uno board consists of ATmega328P microcontroller for data processing. A $4 \mathrm{~K} \Omega$ potentiometer is used for measuring the elbow joint angle $(\beta)$ in terms of change in resistance. The joint parameters are computed indirectly from the value of one sensor outcome. The joint angular velocity $(\omega)$ is calculated from the differentiation of joint angle $(\beta)$ with reference to the timeframe. Joint frequency of elbow is evaluated from angular velocity. The position of the concentric slider $\left(x_{s 2}\right)$ is measured from the relationship between $\beta$ and $x_{s 2}$, Eq. (8). A reflective optical sensor (Model: GP2Y0A41SK0F, (Operating voltage: $5 \mathrm{~V}$, Weight: $0.003 \mathrm{~kg}$ )) is used to measure the position of the nut slider $(x)$. There are other non-contact type of displacement sensors such as inductive, proximity or ultrasonic, however considering the cost and linearity, optical sensor is selected. The range of the optical sensor is 4 to $30 \mathrm{~mm}$. The joint torque is evaluated from other parameters such as from computed joint torque, assistive torque and joint stiffness. All these joint parameters depend on the position of the nut slider $(x)$, concentric slider $\left(x_{s 2}\right)$ and measured joint angle ( $\beta$ ), as shown in Eq. (20). All sensors' output pins are connected to the analog inputs of Arduino board from pin A0 to A1. The DC motor is connected to Arduino using an Arduino compatible motor driver (Model: DFR0225:V2, Operating voltage: $12 \mathrm{~V}$, Weight: $0.080 \mathrm{~kg}$ ). It consists of a L298 to enhance the current level for driving the motor. The maximum speed of the DC motor is $50 \mathrm{rpm}$, however due to the facility of PWM signal, the speed of the motor can be controlled. If those joint parameters cross a particular limit set for the recovery mode, microcontroller sends a command to the motor to change the position of nut slider for changing the rehabilitation mode. In case of unavailability of sensors data, users can fix it manually by varying the nut slider position. The use of software is kept to a minimum just to operate the mechanism but the device is mechanically driven.

$\beta=$ Value of potentiometer

$$
\omega=\frac{d \beta}{d t}
$$

$$
f=\frac{\omega}{2 \pi}
$$

$\tau_{p}=\left(M g L_{1} \cos \beta\right)-\left(\frac{K_{2} r\left(x_{s 2}-x_{s 2}^{\prime}\right) \cos (\alpha-\beta)}{\sin \alpha}\right)+$

$K^{\prime} \beta$ 


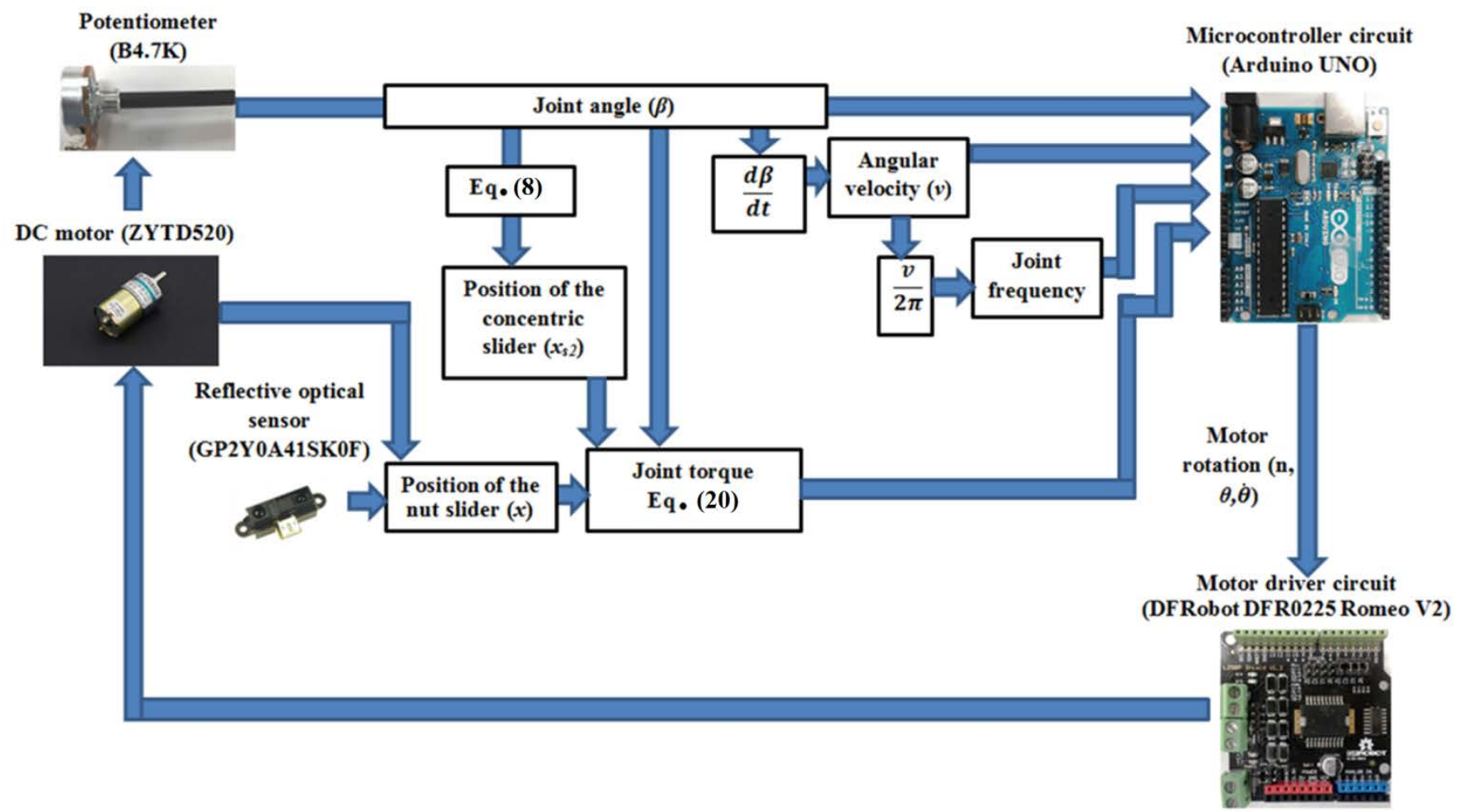

Figure 17. Components of the electronic circuit to drive the motor

\section{Results}

The joint torque of a subject (approximately $1 \mathrm{~kg}$ forearm) is evaluated using the exoskeleton. In the motor controlled mode, the joint angle is changed due to the pulling force. In the assistive mode, user can control the joint with variable assistive force from the exoskeleton, as a result the maximum joint torque is reduced from $1.175 \mathrm{Nm}$ to $1.138 \mathrm{Nm}$ because the assistance of the exoskeleton is increased by changing the position of the nut-slider in the mid region (Fig. 18).

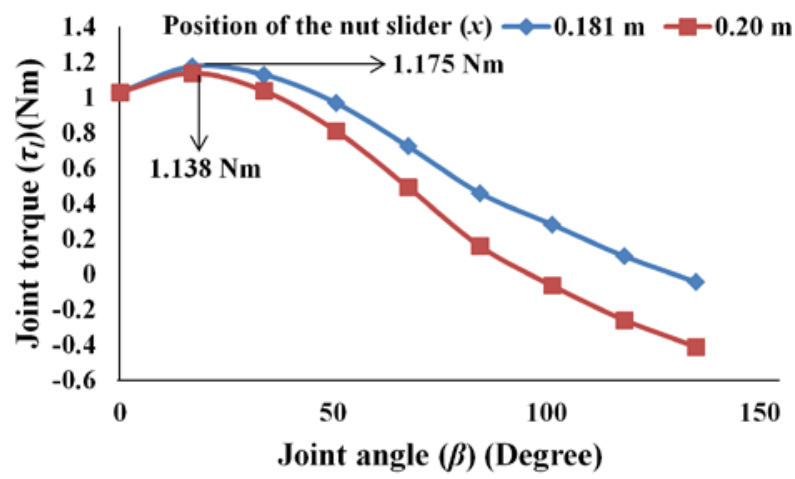

Figure 18. Reduction of joint torque in assistive mode
In the third region, the resistive force provided by the exoskeleton is increased due to the backward movement of the nut-slider, thus the maximum value of the user's joint torque is increased from $1.175 \mathrm{Nm}$ to $1.232 \mathrm{Nm}$ (Fig. 19). The joint torque of individual user can be varied, so is the joint angle and velocity because these joint parameters depend on their own effort.

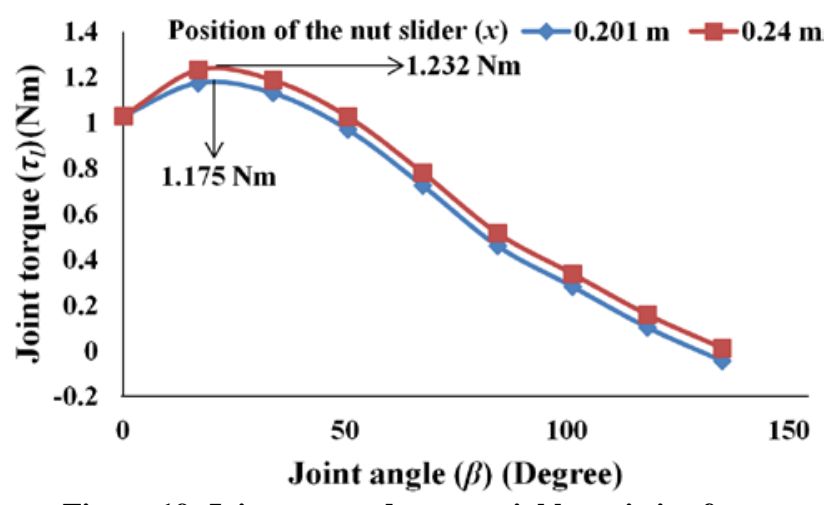

Figure 19. Joint torque due to variable resistive force

In this exoskeleton the switching of rehabilitation modes is decided as per some predefined joint 
values. Motor speed controls the angular velocity in the first rehabilitation mode. The angular velocity of the joint is determined by physiotherapist as per the standard procedure of therapy. Joint angular velocity of the movement can be measured in terms of joint frequency (a full clockwise and anticlockwise rotation). Fig. 20 shows the region wise variation of joint parameters and switching condition. The switching value of joint torque is decided as per the forearm weight of the user and the springs used in the exoskeleton.

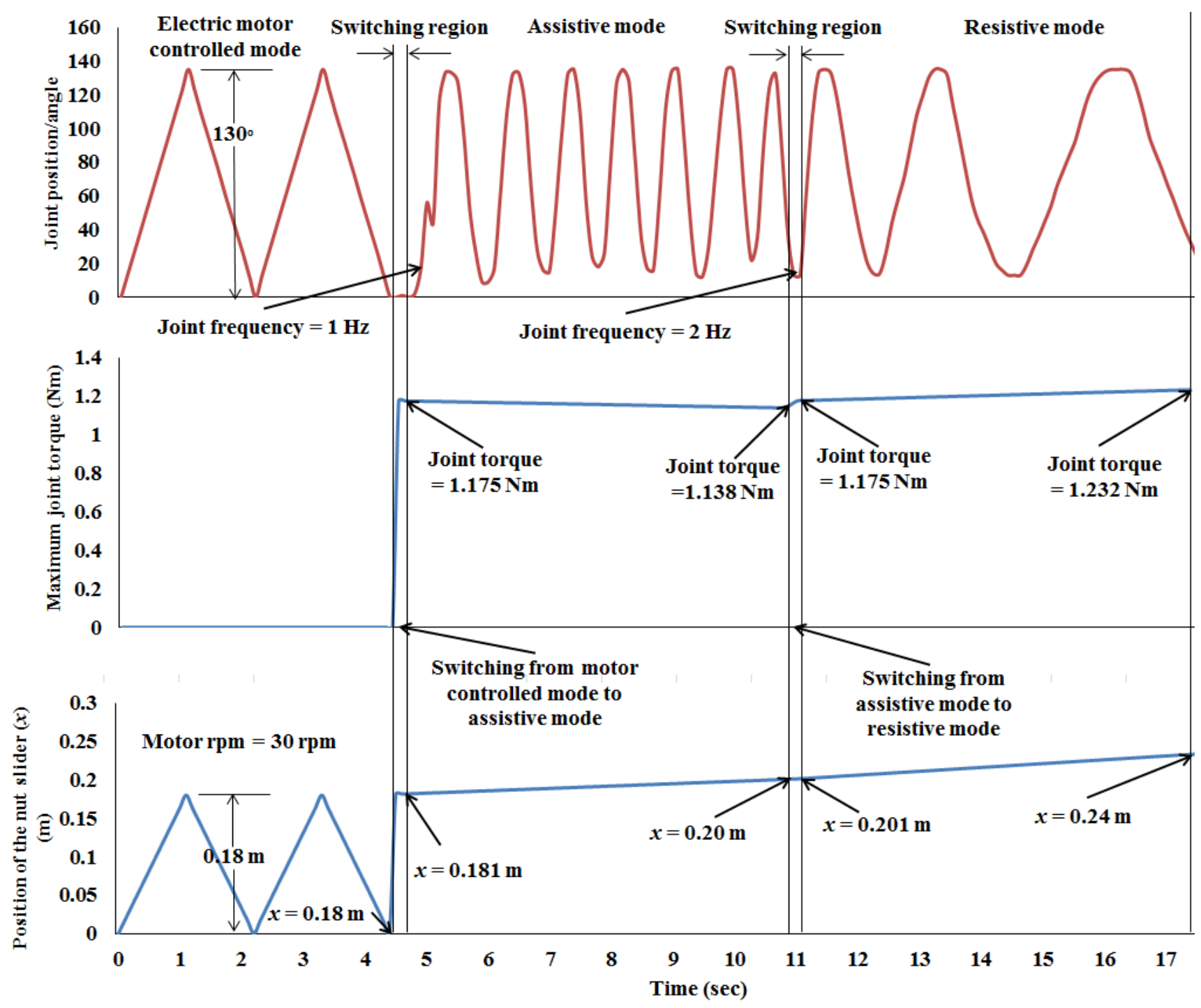

Figure 20. Switching of rehabilitation modes for different position of the nut slider

Due to the reciprocating motion of the nut slider between 0 and $0.18 \mathrm{~m}$, the rotation of elbow joint is controlled by the fixed value of motor speed which can be changed depending on requirement. As long as the nut slider maintains its movement in the first region, the motor controlled joint rotation takes place. This part of the region is used for the post-stroke patients enduring their early stage where their strength is too low to start the joint movement. Consequently, the exoskeleton needs full hold on user's joint movement. The switching region between motor controlled and assistive mode is sufficiently small (approximately $0.001 \mathrm{~m}$ ) without interrupting the operation of the exoskeleton. In this region, the control of joint movement is transferred from 
motor to user. The user can start the joint movement in assistive mode with their normal strength. That's why the middle region is configured to provide support to users. The joint condition is improved with steady and intense rehabilitation process; therefore, patients can produce small joint movement. At the beginning, minimum assistive force is involved with the user's joint movement when the nut slider is positioned at $0.181 \mathrm{~m}$. As it moves backward up to $0.20 \mathrm{~m}$, the assistive force increases and joint torque of the user is gradually decreased (from $1.175 \mathrm{Nm}$ to $1.138 \mathrm{Nm}$ ). In this exoskeleton, the boundary condition of the starting and ending phase of the assistive region is configured as per the joint rotational frequency level of 1-2 Hz. In normal condition, the user can move joint at $1 \mathrm{~Hz}$ which can be improved with time due to the increment at assistance provided by the exoskeleton. User will be promoted to the last stage of recovery if they are able to cross the frequency limit of $2 \mathrm{~Hz}$. As discussed in the design section, the last region is used to enable the users to do joint movements with enhanced joint stiffness needed in the last stage of recovery. This technique creates more resistive force during exercises. The switching region between assistive and resistive mode is also approximately $0.001 \mathrm{~m}$. At the starting of resistive mode, the extended spring $\left(\mathrm{S}_{2}\right)$ restores to its original condition, therefore the joint torque of the user is the same as the beginning phase of assistive mode (1.175 $\mathrm{Nm}$ ). As the nut slider translates backward in this region from $0.201 \mathrm{~m}$ to $0.24 \mathrm{~m}$, the required joint torque increases from $1.175 \mathrm{Nm}$ to $1.232 \mathrm{Nm}$ however the joint frequency decreases due to more resistive force.

\section{Discussion and conclusions}

A mechanical elbow exoskeleton has been developed for measuring and controlling the joint parameters during post-stroke rehabilitation. After analysing the results, it is shown that the exercises related to three stages of rehabilitation are feasible with the developed exoskeleton. The mechanical requirements related to actuation system of the exoskeleton are different depending on the situation whether the exoskeleton provides the exercise or user controls the rehabilitation process. In motor controlled mode, the joint angle and speed of the joint movement can be increased with time by changing the parameter of motor rotation. The mechanism of the exoskeleton can change the amount of assistive and resistive force as per the user's need. However, all these modes of exercises can be controlled by changing the region of operation. The aim of assistive and resistive rehabilitation is to improve the joint effort either by decreasing the assistive force or increasing the resistive force over time. The switching of rehabilitation mode takes place automatically due to the stiffness property of springs in the exoskeleton. It is also possible to change the rehabilitation mode by just changing the position of the nut slider unlike the torque control of joint based motor; therefore, the device is mechanically tunable to users' need. The developed device is functional and the size and weight of the system can be further reduced with suitable materials. The paper describes an adaptive rehabilitation therapy by integrating three types of rehabilitation in a single exoskeleton. Three joint parameters: joint angle, angular velocity and joint torque are incorporated into the exoskeleton to optimally provide post-stroke rehabilitation. Based on the requirements, any rehabilitation mode can be selected. The developed exoskeleton provides a mechanism based solution which largely removes the dependency of biosensors. The exoskeleton is operated using a single motor. The switching mechanism between motor controlled mode and assistive mode works safely because the control of the joint motion is not done by the motor. In assistive and resistive rehabilitation, since springs are used to provide the required forces without using any extra energy sources, this reduces the power consumption as well as the size of the energy source required. In the developed prototype, the joint frequency of switching between motor control to assistive mode is fixed at $1 \mathrm{~Hz}$ whereas the boundary value between assistive mode and resistive mode is fixed at $2 \mathrm{~Hz}$. Also the joint angle, velocity and static torque of 
the exoskeleton in different rehabilitation modes are decided based on forearm weight and mechanical property of springs. However, those switching parameters can be changed for different user as recommended by physiotherapist.

\section{References}

1. Thrift AG, Thayabaranathan T, Howard G, et al. Global stroke statistics. International Journal of Stroke. 2017; 12: 13-32.

2. Lo HS and Xie SQ. Exoskeleton robots for upper-limb rehabilitation: State of the art and future prospects. Medical engineering \& physics. 2012; 34: 261-8.

3. Krebs HI, Ferraro M, Buerger SP, et al. Rehabilitation robotics: pilot trial of a spatial extension for MIT-Manus. Journal of neuroengineering and rehabilitation. 2004; $1: 5$.

4. Proietti T, Crocher V, Roby-Brami A and Jarrassé N. Upper-limb robotic exoskeletons for neurorehabilitation: a review on control strategies. IEEE reviews in biomedical engineering. 2016; 9: 4-14.

5. Pineda-Rico Z, de Lucio JAS, Martinez Lopez FJ and Cruz P. 2121. Design of an exoskeleton for upper limb robot-assisted rehabilitation based on co-simulation. Journal of Vibroengineering. 2016; 18.

6. Chonnaparamutt W and Supsi W. SEFRE: Semiexoskeleton Rehabilitation System. Applied Bionics and Biomechanics. 2016; 2016.

7. Peternel L, Noda T, Petrič T, Ude A, Morimoto $\mathrm{J}$ and Babič J. Adaptive control of exoskeleton robots for periodic assistive behaviours based on EMG feedback minimisation. PloS one. 2016; 11: e0148942.

8. Bhadane M, Liu J, Rymer WZ, Zhou P and Li S. Re-evaluation of EMG-torque relation in chronic stroke using linear electrode array EMG recordings. Scientific Reports. 2016; 6: 28957.

9. Cesqui B, Tropea P, Micera $S$ and Krebs HI. EMG-based pattern recognition approach in post stroke robot-aided rehabilitation: a feasibility study. Journal of neuroengineering and rehabilitation. 2013; 10: 75.

10. Koyas E, Hocaoglu E, Patoglu V and Cetin M. Detection of intention level in response to task difficulty from EEG signals. 2013 IEEE International Workshop on Machine Learning for Signal Processing. IEEE, 2013, p. 1-6.

11. Marchal-Crespo L and Reinkensmeyer DJ. Review of control strategies for robotic movement training after neurologic injury. Journal of neuroengineering and rehabilitation. 2009; 6: 20.

12. Ibrahim I, Berger W, Trippel M and Dietz V. Stretch-induced electromyographic activity and torque in spastic elbow muscles: differential modulation of reflex activity in passive and active motor tasks. Brain. 1993; 116: 971-89.

13. Chiri A, Giovacchini F, Vitiello N, et al. HANDEXOS: Towards an exoskeleton device for the rehabilitation of the hand. IEEE/RSJ International Conference on Intelligent Robots and Systems. IEEE, 2009, p. 1106-11.

14. Herder JL, Vrijlandt N, Antonides T, Cloosterman M and Mastenbroek PL. Principle and design of a mobile arm support for people with muscular weakness. Journal of rehabilitation research and development. 2006; 43: 591.

15. Manna SK, Dubey, V.N. Upper arm exoskeleton -what specifications will meet users' acceptability? . In: Fisher DG, (ed.). Robotics: New Research Nova Science Publisher, 2016, p. 123-69.

16. Manna SK and Dubey VN. A novel hardware approach to integrating active and passive rehabilitation in a single exoskeleton. International Symposium on Medical Robotics. IEEE, 2018, p. 1-6. 